Article

\title{
Some Ostrowski type integral inequalities via generalized harmonic convex functions
}

\author{
Muhammad Tariq ${ }^{1}$ and Saad Ihsan Butt ${ }^{1, *}$ \\ 1 Department of Mathematics, COMSATS University Islamabad, Lahore Campus, Pakistan. \\ * Correspondence: saadihsanbutt@cuilahore.edu.pk \\ Communicated by: Waqas Nazeer \\ Received: 25 November 2020; Accepted: 21 April 2021; Published: 18 May 2021.
}

\begin{abstract}
In this paper, we aim to introduce a new notion of convex functions namely the harmonic s-type convex functions. The refinements of Ostrowski type inequality are investigated which are the generalized and extended variants of the previously known results for harmonic convex functions.
\end{abstract}

Keywords: Hermite-Hadamard inequality; Hölder's inequality; Harmonic convex functions; Harmonic s-type convex function.

MSC: 26A51; 26A33; 26D07; 26D10; 26D15.

\section{Introduction}

C urrently the theory of convexity is a hot filed of research due to its applications in optimization theory. This theory also provides the solution to many problems which arise in applied and pure mathematics. This theory plays a remarkable role in the study of linear and non-linear programming problems. In past years, this theory attracts many mathematicians, see [1-3].

In mathematics, the idea of convexity is based on the theory of inequalities. The current perspective on the term inequalities require a robust and interconnection between geometry and analysis, which makes the reader shear a sense of exhilaration and exultation. For the applications interested readers can see [4,5].

In 1938, A. Ostrowski established an inequality namely Ostrowski inequality. Mathematically Ostrowski inequality [6] is given by, let $\Psi: \mathbb{A} \subseteq \mathbb{R} \rightarrow \mathbb{R}$ is an differential function on $\mathbb{A}^{\circ}$ and $\wp_{1}, \wp_{2} \in \mathbb{A}^{\circ}$ with $\wp_{1}<\wp_{2}$. If $\left|\Psi^{\prime}(x)\right| \leqslant M, \forall x \in\left[\wp_{1}, \wp_{2}\right]$, then we attain the following inequality

$$
\left|\Psi(x)-\frac{1}{\wp_{2}-\wp_{1}} \int_{\wp_{1}}^{\wp_{2}} \Psi(u) d u\right| \leqslant M\left(\wp_{2}-\wp_{1}\right)\left[\frac{1}{4}-\frac{x-\left(\frac{\wp_{1}+\wp_{2}}{2}\right)^{2}}{\left(\wp_{2}-\wp_{1}\right)^{2}}\right], \quad \forall x \in\left(\wp_{1}, \wp_{2}\right) .
$$

This inequality has a great contribution and various applications in probability, numerical integration, and mathematical analysis. The $1^{\text {st }}$ time extension and multivariate of this inequality was investigated by Milovanović and Peçarić in [7] and Milovanović in [8] respectively. The Dragomir and Wang [9] explored an inequality of Ostrowski-Grüss type. From the last few decades, due to the effectiveness and applications, many authors generalized the Ostrowski's inequality, see [10-12].

In this paper, the refinements of Ostrowski type inequalities for s-type convex functions are investigated, which are the generalized and extended variants of the previously known results.

\section{Preliminaries}

A function $\Psi: \mathbb{A} \rightarrow \mathbb{R}$ is called convex [13], if the following inequality

$$
\Psi\left(\kappa \wp_{1}+(1-\kappa) \wp_{2}\right) \leqslant \kappa \Psi\left(\wp_{1}\right)+(1-\kappa) \Psi\left(\wp_{2}\right)
$$

holds for all $\wp_{1}, \wp_{2} \in \mathbb{A}$ and $\kappa \in[0,1]$.

Harmonic means balance the weights of each data point which is the most relevant and pertinent measure for rates and ratios. The harmonic convex set is always defined by harmonic mean which was introduced by Shi and Zhang [14]. İşcan [15] defined the harmonic convex function. 
Definition 1. [15] A function $\Psi: \mathbb{A} \subseteq(0,+\infty) \rightarrow \mathbb{R}$ is called harmonic convex, if

$$
\Psi\left(\frac{\wp_{1} \wp_{2}}{\kappa \wp_{2}+(1-\kappa) \wp_{1}}\right) \leqslant \kappa \Psi\left(\wp_{1}\right)+(1-\kappa) \Psi\left(\wp_{2}\right)
$$

holds for all $\wp_{1}, \wp_{2} \in \mathbb{A}$ and $\kappa \in[0,1]$.

Theorem 1. [15] Let $\Psi: \mathbb{A} \subseteq(0,+\infty) \rightarrow \mathbb{R}$ be a harmonically convex function. If $\Psi \in L\left[\wp_{1}, \wp_{2}\right]$ for all $\wp_{1}, \wp_{2} \in \mathbb{A}$ with $\wp_{1}<\wp_{2}$, then

$$
\Psi\left(\frac{2 \wp_{1} \wp_{2}}{\wp_{1}+\wp_{2}}\right) \leqslant \frac{\wp_{1} \wp_{2}}{\wp_{2}-\wp_{1}} \int_{\wp_{1}}^{\wp_{2}} \frac{\Psi(x)}{x^{2}} d x \leqslant \frac{\Psi\left(\wp_{1}\right)+\Psi\left(\wp_{2}\right)}{2} .
$$

Recently Rashid et al., [16] introduced s-type convex function as follows:

Definition 2. [16] A function $\Psi: \mathbb{A} \rightarrow \mathbb{R}$ is said to be $s$-type convex function, if

$$
\Psi\left(\kappa \wp_{1}+(1-\kappa) \wp_{2}\right) \leqslant[1-s(1-\kappa)] \Psi\left(\wp_{1}\right)+[1-s \kappa] \Psi\left(\wp_{2}\right)
$$

holds $\forall \wp_{1}, \wp_{2} \in \mathbb{A}, \kappa \in[0,1]$ and $s \in[0,1]$.

The term "hypergeometric function" sometimes refers to the generalized hypergeometric function. In 1949, Arnold Sommerfeld [17] introduced the hypergeometric function by the differential equation. In 1960, Kratzer and Franz determined the hypergeometric function in the support of hypergeometric series. In 1963, Lebedev investigated the hypergeometric function through the $\Gamma$-function and its logarithmic derivative. In 2006, Françoise et al., [18] introduced the Gaüss hypergeometric function defined for $|z|<1$ by the hypergeometric series. This function is often represented by $F(a, b ; c ; z)$. Mathematically hypergeometric function is given as [19]:

$$
{ }_{2} F_{1}(a, b ; c ; z)=\frac{1}{\beta(b, c-b)} \int_{0}^{1} \kappa^{b-1}(1-\kappa)^{c-b-1}(1-z \kappa)^{-a} d \kappa, c>b>0,|z|<1 .
$$

This functions have a wide range of applications in partitions, combinatorial identities, cylinder and spherical problems, finite vector spaces, number theory, and physics.

Motivated by ongoing research activities in this direction, first of all in Section 3, we will explore the concept of harmonic s-type convex function. In addition, some examples in the manner of this newly introduced definition are elaborated. Furthermore, in Section 4, we investigate some refinements of the Ostrowski type inequality. Finally, we will give a conclusion.

\section{Generalized harmonic convex function and its properties}

The principal objective of this section is to define the new class of convex functions which are called harmonic s-type convex functions.

Definition 3. A function $\Psi: \mathbb{A} \subseteq(0,+\infty) \rightarrow[0,+\infty)$ is called harmonic s-type convex, if

$$
\Psi\left(\frac{\wp_{1} \wp_{2}}{\kappa \wp_{2}+(1-\kappa) \wp_{1}}\right) \leqslant[1-s(1-\kappa)] \Psi\left(\wp_{1}\right)+[1-s \kappa] \Psi\left(\wp_{2}\right)
$$

holds for every $\wp_{1}, \wp_{2} \in \mathbb{A}, \kappa \in[0,1]$ and $s \in[0,1]$.

Remark 1. Taking $s=1$ in Definition 3, we attain the definition of harmonically convex function which is defined by İşcan [15].

Lemma 1. The inequalities $[1-s(1-\kappa)] \geqslant \kappa$ and $[1-s \kappa] \geqslant(1-\kappa)$ hold $\forall s \in[0,1]$ and $\kappa \in[0,1]$.

Proposition 1. Every harmonic convex function on $\mathbb{A} \subset(0,+\infty)$ is harmonic s-type convex function. 
Proof. From $\kappa \leqslant[1-s(1-\kappa)]$ and $(1-\kappa) \leqslant[1-s \kappa]$ for all $\kappa \in[0,1]$ and $s \in[0,1]$ and applying the property of harmonic convex function, we have

$$
\Psi\left[\frac{\wp_{1} \wp_{2}}{\kappa \wp_{2}+(1-\kappa) \wp_{1}}\right] \leqslant \kappa \Psi\left(\wp_{1}\right)+(1-\kappa) \Psi\left(\wp_{2}\right) \leqslant[1-s(1-\kappa)] \Psi\left(\wp_{1}\right)+[1-s \kappa] \Psi\left(\wp_{2}\right) .
$$

Proposition 2. Every harmonic s-type convex function is harmonic h-convex function with $h(\kappa)=[1-s(1-\kappa)]$.

Proof. Applying the property of harmonic s-type convex function and using the condition $h(\kappa)=[1-s(1-$ $\kappa)$ ], we have

$$
\begin{aligned}
\Psi\left[\frac{\wp_{1} \wp_{2}}{\kappa \wp_{2}+(1-\kappa) \wp_{1}}\right] & \leqslant[1-s(1-\kappa)] \Psi\left(\wp_{1}\right)+[1-s \kappa] \Psi\left(\wp_{2}\right) \\
& \leqslant h(\kappa) \Psi\left(\wp_{1}\right)+h(1-\kappa) \Psi\left(\wp_{2}\right) .
\end{aligned}
$$

Now we give some examples.

Example 1. As $\Psi(\varsigma)=e^{\varsigma}$ is an increasing convex function so it is harmonically convex function (see [20]). By using Proposition 1 , it is a harmonic s-type convex function.

Example 2. As $\Psi(\varsigma)=\ln \varsigma$ is an increasing convex function so it is harmonically convex function (see [20]). By using Proposition 1, it is a harmonic s-type convex function.

\section{Refinements of Ostrowski type inequality via harmonic s-type convex functions}

In this section, we are going to attain some refinements of Ostrowski type inequality by the way of harmonic s-type convex function. Throughout this section, diff mapp means differentiable mapping. In order to obtain the results, we need the following lemma:

Lemma 2. [21] Let $\Psi: \mathbb{A} \subseteq \mathbb{R} \backslash\{0\} \rightarrow \mathbb{R}$ be a diff mapp on $\mathbb{A}^{0}$, where $\wp_{1}, \wp_{2} \in \mathbb{A}$ with $\wp_{1}<\wp_{2}$. If $\Psi^{\prime} \in L\left[\wp_{1}, \wp_{2}\right]$, then

$$
\begin{aligned}
\Psi(x)-\frac{\wp_{1} \wp_{2}}{\wp_{2}-\wp_{1}} \int_{\wp_{1}}^{\wp_{2}} \frac{\Psi(u)}{u^{2}} d u= & \frac{\wp_{1} \wp_{2}}{\wp_{2}-\wp_{1}}\left\{\left(x-\wp_{1}\right)^{2} \int_{0}^{1} \frac{\kappa}{\left(\kappa \wp_{1}+(1-\kappa) x\right)^{2}} \Psi^{\prime}\left(\frac{\wp_{1} x}{\left(\kappa \wp_{1}+(1-\kappa) x\right)}\right) d \kappa\right. \\
& \left.-\left(\wp_{2}-x\right)^{2} \int_{0}^{1} \frac{\kappa}{\left(\kappa \wp_{2}+(1-\kappa) x\right)^{2}} \Psi^{\prime}\left(\frac{\wp_{2} x}{\left(\kappa \wp_{2}+(1-\kappa) x\right)}\right) d \kappa\right\}
\end{aligned}
$$

for each $x \in\left[\wp_{1}, \wp_{2}\right]$.

Theorem 2. Let $\Psi: \mathbb{A}=\left[\wp_{1}, \wp_{2}\right] \subseteq(0,+\infty) \rightarrow \mathbb{R}$ be a diff mapp on $\mathbb{A}^{0}$, where $\wp_{1}, \wp_{2} \in \mathbb{A}$ with $\wp_{1}<\wp_{2}$ and $\Psi^{\prime} \in L\left[\wp_{1}, \wp_{2}\right]$. If the mapping $\left|\Psi^{\prime}\right|^{l}$ is harmonic s-type convex for $l \geqslant 1$ and $s \in[0,1]$, then

$$
\begin{aligned}
\mid \Psi(x) & -\frac{\wp_{1} \wp_{2}}{\wp_{2}-\wp_{1}} \int_{\wp_{1}}^{\wp_{2}} \frac{\Psi(u)}{u^{2}} d u \mid \\
& \leqslant \frac{\wp_{1} \wp_{2}}{\wp_{2}-\wp_{1}}\left\{\left(x-\wp_{1}\right)^{2}\left(\Lambda_{1}\left|\Psi^{\prime}(x)\right|^{l}+\Lambda_{2}\left|\Psi^{\prime}\left(\wp_{1}\right)\right|^{l}\right)^{\frac{1}{l}}+\left(\wp_{2}-x\right)^{2}\left(\Lambda_{3}\left|\Psi^{\prime}(x)\right|^{l}+\Lambda_{4}\left|\Psi^{\prime}\left(\wp_{2}\right)\right|^{l}\right)^{\frac{1}{l}}\right\},
\end{aligned}
$$

where

$$
\begin{aligned}
& \Lambda_{1}=\frac{\beta(l+1,1)}{x^{2 l}}{ }_{2} F_{1}\left(2 l, l+1 ; l+2 ; 1-\frac{\wp_{1}}{x}\right)-s \frac{\beta(l+1,2)}{x^{2 l}}{ }_{2} F_{1}\left(2 l, l+1 ; l+2 ; 1-\frac{\wp_{1}}{x}\right), \\
& \Lambda_{2}=\frac{\beta(l+1,1)}{x^{2 l}}{ }_{2} F_{1}\left(2 l, l+1 ; l+2 ; 1-\frac{\wp_{1}}{x}\right)-s \frac{\beta(l+2,1)}{x^{2 l}}{ }_{2} F_{1}\left(2 l, l+2 ; l+3 ; 1-\frac{\wp_{1}}{x}\right), \\
& \Lambda_{3}=\frac{\beta(l+1,1)}{x^{2 l}}{ }_{2} F_{1}\left(2 l, l+1 ; l+2 ; 1-\frac{\wp_{2}}{x}\right)-s \frac{\beta(l+1,2)}{x^{2 l}}{ }_{2} F_{1}\left(2 l, l+1 ; l+3 ; 1-\frac{\wp_{2}}{x}\right),
\end{aligned}
$$


and

$$
\Lambda_{4}=\frac{\beta(l+1,1)}{x^{2 l}}{ }_{2} F_{1}\left(2 l, l+1 ; l+2 ; 1-\frac{\wp_{2}}{x}\right)-s \frac{\beta(l+2,1)}{x^{2 l}}{ }_{2} F_{1}\left(2 l, l+2 ; l+3 ; 1-\frac{\wp_{2}}{x}\right) .
$$

Proof. Applying Lemma 2, properties of modulus, power mean inequality and the property of harmonic s-type convex function of $\left|\Psi^{\prime}\right|^{l}$, we have

$$
\begin{aligned}
& \left|\Psi(x)-\frac{\wp_{1} \wp_{2}}{\wp_{2}-\wp_{1}} \int_{\wp_{1}}^{\wp_{2}} \Psi(u) d u\right| \\
& \leqslant \frac{\wp_{1} \wp_{2}}{\wp_{2}-\wp_{1}}\left\{\left(x-\wp_{1}\right)^{2} \int_{0}^{1} \frac{\kappa}{\left(\kappa \wp_{1}+(1-\kappa) x\right)^{2}}\left|\Psi^{\prime}\left(\frac{\wp_{1} x}{\left(\kappa \wp_{1}+(1-\kappa) x\right)}\right)\right| d \kappa\right. \\
& \left.+\left(\wp_{2}-x\right)^{2} \int_{0}^{1} \frac{\kappa}{\left(\kappa \wp_{2}+(1-\kappa) x\right)^{2}}\left|\Psi^{\prime}\left(\frac{\wp_{2} x}{\left(\kappa \wp_{2}+(1-\kappa) x\right)}\right)\right| d \kappa\right\} \\
& \leqslant \frac{\wp_{1} \wp_{2}\left(x-\wp_{1}\right)^{2}}{\wp_{2}-\wp_{1}}\left(\int_{0}^{1} 1 d \kappa\right)^{1-\frac{1}{l}}\left(\int_{0}^{1} \frac{\kappa^{l}}{\left(\kappa \wp_{1}+(1-\kappa) x\right)^{2 l}}\left|\Psi^{\prime}\left(\frac{\wp_{1} x}{\left(\kappa \wp_{1}+(1-\kappa) x\right)}\right)\right|^{l} d \kappa\right)^{\frac{1}{l}} \\
& +\frac{\wp_{1} \wp_{2}\left(\wp_{2}-x\right)^{2}}{\wp_{2}-\wp_{1}}\left(\int_{0}^{1} 1 d \kappa\right)^{1-\frac{1}{l}}\left(\int_{0}^{1} \frac{\kappa^{l}}{\left(\kappa \wp_{2}+(1-\kappa) x\right)^{2 l}}\left|\Psi^{\prime}\left(\frac{\wp_{2} x}{\left(\kappa \wp_{2}+(1-\kappa) x\right)}\right)\right|^{l} d \kappa\right)^{\frac{1}{l}} \\
& \leqslant \frac{\wp_{1} \wp_{2}\left(x-\wp_{1}\right)^{2}}{\wp_{2}-\wp_{1}}\left(\int_{0}^{1} \frac{\kappa^{l}\left[[1-s(1-\kappa)]\left|\Psi^{\prime}(x)\right|^{l}+[1-s \kappa]\left|\Psi^{\prime}\left(\wp_{1}\right)\right|^{l}\right]}{\left(\kappa \wp_{1}+(1-\kappa) x\right)^{2 l}} d \kappa\right)^{\frac{1}{T}} \\
& +\frac{\wp_{1} \wp_{2}\left(\wp_{2}-x\right)^{2}}{\wp_{2}-\wp_{1}}\left(\int_{0}^{1} \frac{\kappa^{l}\left[[1-s(1-\kappa)]\left|\Psi^{\prime}(x)\right|^{l}+[1-s \kappa]\left|\Psi^{\prime}\left(\wp_{2}\right)\right|^{l}\right]}{\left(\kappa \wp_{2}+(1-\kappa) x\right)^{2 l}} d \kappa\right)^{\frac{1}{T}} \\
& \leqslant \frac{\wp_{1} \wp_{2}\left(x-\wp_{1}\right)^{2}}{\wp_{2}-\wp_{1}}\left(\int_{0}^{1} \frac{\kappa^{l}[1-s(1-\kappa)]}{\left(\kappa \wp_{1}+(1-\kappa) x\right)^{2 l}} d \kappa\left|\Psi^{\prime}(x)\right|^{l}+\int_{0}^{1} \frac{\left.\kappa^{l}[1-(s \kappa))\right]}{\left(\kappa \wp_{1}+(1-\kappa) x\right)^{2 l}} d \kappa\left|\Psi^{\prime}\left(\wp_{1}\right)\right|^{l}\right)^{\frac{1}{l}} \\
& +\frac{\wp_{1} \wp_{2}\left(\wp_{2}-x\right)^{2}}{\wp_{2}-\wp_{1}}\left(\int_{0}^{1} \frac{\kappa^{l}[1-s(1-\kappa)]}{\left(\kappa \wp_{2}+(1-\kappa) x\right)^{2 l}} d \kappa\left|\Psi^{\prime}(x)\right|^{l}+\int_{0}^{1} \frac{\left.\kappa^{l}[1-(s \kappa))\right]}{\left(\kappa \wp_{2}+(1-\kappa) x\right)^{2 l}} d \kappa\left|\Psi^{\prime}\left(\wp_{2}\right)\right|^{l}\right)^{\frac{1}{l}} \\
& \leqslant \frac{\wp_{1} \wp_{2}}{\wp_{2}-\wp_{1}}\left\{\left(x-\wp_{1}\right)^{2}\left(\Lambda_{1}\left|\Psi^{\prime}(x)\right|^{l}+\Lambda_{2}\left|\Psi^{\prime}\left(\wp_{1}\right)\right|^{l}\right)^{\frac{1}{l}}+\left(\wp_{2}-x\right)^{2}\left(\Lambda_{3}\left|\Psi^{\prime}(x)\right|^{l}+\Lambda_{4}\left|\Psi^{\prime}\left(\wp_{2}\right)\right|^{l}\right)^{\frac{1}{l}}\right\} \text {. }
\end{aligned}
$$

The proof is complete.

Corollary 1. In Theorem 2, taking $\left|\Psi^{\prime}(x)\right| \leqslant M, \forall x \in\left[\wp_{1}, \wp_{2}\right]$, we attain the following inequality

$$
\left|\Psi(x)-\frac{\wp_{1} \wp_{2}}{\wp_{2}-\wp_{1}} \int_{\wp_{1}}^{\wp_{2}} \frac{\Psi(u)}{u^{2}} d u\right| \leqslant \frac{\wp_{1} \wp_{2}}{\wp_{2}-\wp_{1}} M\left\{\left(x-\wp_{1}\right)^{2}\left(\Lambda_{1}+\Lambda_{2}\right)^{\frac{1}{T}}+\left(\wp_{2}-x\right)^{2}\left(\Lambda_{3}+\Lambda_{4}\right)^{\frac{1}{T}}\right\} .
$$

Theorem 3. Let $\Psi: \mathbb{A}=\left[\wp_{1}, \wp_{2}\right] \subseteq(0,+\infty) \rightarrow \mathbb{R}$ be a diff map on $\mathbb{A}^{0}$, where $\wp_{1}, \wp_{2} \in \mathbb{A}$ with $\wp_{1}<\wp_{2}$ and $\Psi^{\prime} \in L\left[\wp_{1}, \wp_{2}\right]$. If the mapping $\left|\Psi^{\prime}\right|^{l}$ is harmonic $s$-type convex for $l \geqslant 1$ and $s \in[0,1]$ then for all $x \in\left[\wp_{1}, \wp_{2}\right]$, one has

$$
\begin{aligned}
\mid \Psi(x) & -\frac{\wp_{1} \wp_{2}}{\wp_{2}-\wp_{1}} \int_{\wp_{1}}^{\wp_{2}} \frac{\Psi(u)}{u^{2}} d u \mid \\
& \leqslant \frac{\wp_{1} \wp_{2}}{\wp_{2}-\wp_{1}}\left(\frac{1}{2}\right)^{1-\frac{1}{l}}\left\{\left(x-\wp_{1}\right)^{2}\left(\Lambda_{5}\left|\Psi^{\prime}(x)\right|^{l}+\Lambda_{6}\left|\Psi^{\prime}\left(\wp_{1}\right)\right|^{l}\right)^{\frac{1}{l}}+\left(\wp_{2}-x\right)^{2}\left(\Lambda_{7}\left|\Psi^{\prime}(x)\right|^{l}+\Lambda_{8}\left|\Psi^{\prime}\left(\wp_{2}\right)\right|^{l}\right)^{\frac{1}{l}}\right\}
\end{aligned}
$$

where

and

$$
\begin{aligned}
& \Lambda_{5}=\frac{\beta(2,1)}{x^{2 l}}{ }_{2} F_{1}\left(2 l, 2 ; 3 ; 1-\frac{\wp_{1}}{x}\right)-s \frac{\beta(2,2)}{x^{2 l}}{ }_{2} F_{1}\left(2 l, 2 ; 2 ; 1-\frac{\wp_{1}}{x}\right), \\
& \Lambda_{6}=\frac{\beta(2,1)}{x^{2 l}}{ }_{2} F_{1}\left(2 l, 2 ; 3 ; 1-\frac{\wp_{1}}{x}\right)-s \frac{\beta(3,1)}{x^{2 l}}{ }_{2} F_{1}\left(2 l, 3 ; 4 ; 1-\frac{\wp_{1}}{x}\right), \\
& \Lambda_{7}=\frac{\beta(2,1)}{x^{2 l}}{ }_{2} F_{1}\left(2 l, 2 ; 3 ; 1-\frac{\wp_{2}}{x}\right)-s \frac{\beta(2,2)}{x^{2 l}}{ }_{2} F_{1}\left(2 l, 2 ; 2 ; 1-\frac{\wp_{2}}{x}\right),
\end{aligned}
$$

$$
\Lambda_{8}=\frac{\beta(2,1)}{x^{2 l}}{ }_{2} F_{1}\left(2 l, 2 ; 3 ; 1-\frac{\wp_{2}}{x}\right)-s \frac{\beta(3,1)}{x^{2 l}}{ }_{2} F_{1}\left(2 l, 3 ; 4 ; 1-\frac{\wp_{2}}{x}\right) \text {. }
$$


Proof. Applying Lemma 2, properties of modulus, power mean inequality and the property of harmonic s-type convex function of $\left|\Psi^{\prime}\right|^{l}$, we have

$$
\begin{aligned}
& \left|\Psi(x)-\frac{\wp_{1} \wp_{2}}{\wp_{2}-\wp_{1}} \int_{\wp_{1}}^{\wp_{2}} \Psi(u) d u\right| \\
& \leqslant \frac{\wp_{1} \wp_{2}}{\wp_{2}-\wp_{1}}\left\{\left(x-\wp_{1}\right)^{2} \int_{0}^{1} \frac{\kappa}{\left(\kappa \wp_{1}+(1-\kappa) x\right)^{2}}\left|\Psi^{\prime}\left(\frac{\wp_{1} x}{\left(\kappa \wp_{1}+(1-\kappa) x\right)}\right)\right| d \kappa\right. \\
& \left.+\left(\wp_{2}-x\right)^{2} \int_{0}^{1} \frac{\kappa}{\left(\kappa \wp_{2}+(1-\kappa) x\right)^{2}}\left|\Psi^{\prime}\left(\frac{\wp_{2} x}{\left(\kappa \wp_{2}+(1-\kappa) x\right)}\right)\right| d \kappa\right\} \\
& \leqslant \frac{\wp_{1} \wp_{2}\left(x-\wp_{1}\right)^{2}}{\wp_{2}-\wp_{1}}\left(\int_{0}^{1} \kappa d \kappa\right)^{1-\frac{1}{q}}\left(\int_{0}^{1} \frac{\kappa}{\left(\kappa \wp_{1}+(1-\kappa) x\right)^{2 q}}\left|\Psi^{\prime}\left(\frac{\wp_{1} x}{\left(\kappa \wp_{1}+(1-\kappa) x\right)}\right)\right|^{l} d \kappa\right)^{\frac{1}{T}} \\
& +\frac{\wp_{1} \wp_{2}\left(\wp_{2}-x\right)^{2}}{\wp_{2}-\wp_{1}}\left(\int_{0}^{1} \kappa d \kappa\right)^{1-\frac{1}{l}}\left(\int_{0}^{1} \frac{\kappa}{\left(\kappa \wp_{2}+(1-\kappa) x\right)^{2 l}}\left|\Psi^{\prime}\left(\frac{\wp_{2} x}{\left(\kappa \wp_{2}+(1-\kappa) x\right)}\right)\right|^{l} d \kappa\right)^{\frac{1}{l}} \\
& \leqslant \frac{\wp_{1} \wp_{2}\left(x-\wp_{1}\right)^{2}}{\wp_{2}-\wp_{1}}\left(\frac{1}{2}\right)^{1-\frac{1}{l}}\left(\int_{0}^{1} \frac{\kappa\left[[1-s(1-\kappa)]\left|\Psi^{\prime}(x)\right|^{l}+[1-s \kappa]\left|\Psi^{\prime}\left(\wp_{1}\right)\right|^{l}\right]}{\left(\kappa \wp_{1}+(1-\kappa) x\right)^{2 l}} d \kappa\right)^{\frac{1}{l}} \\
& +\frac{\wp_{1} \wp_{2}\left(\wp_{2}-x\right)^{2}}{\wp_{2}-\wp_{1}}\left(\frac{1}{2}\right)^{1-\frac{1}{l}}\left(\int_{0}^{1} \frac{\kappa\left[[1-s(1-\kappa)]\left|\Psi^{\prime}(x)\right|^{l}+[1-s \kappa]\left|\Psi^{\prime}\left(\wp_{2}\right)\right|^{l}\right]}{\left(\kappa \wp_{2}+(1-\kappa) x\right)^{2 l}} d \kappa\right)^{\frac{1}{l}} \\
& \leqslant \frac{\wp_{1} \wp_{2}}{\wp_{2}-\wp_{1}}\left(\frac{1}{2}\right)^{1-\frac{1}{l}}\left\{\left(x-\wp_{1}\right)^{2}\left(\int_{0}^{1} \frac{\kappa[1-s(1-\kappa)]}{\left(\kappa \wp_{1}+(1-\kappa) x\right)^{2 q}} d \kappa\left|\Psi^{\prime}(x)\right|^{q}+\int_{0}^{1} \frac{\kappa[1-(s \kappa))]}{\left(\kappa \wp_{1}+(1-\kappa) x\right)^{2 l}} d \kappa\left|\Psi^{\prime}\left(\wp_{1}\right)\right|^{l}\right)^{\frac{1}{l}}\right. \\
& \left.+\left(\wp_{2}-x\right)^{2}\left(\int_{0}^{1} \frac{\kappa[1-s(1-\kappa)]}{\left(\kappa \wp_{2}+(1-\kappa) x\right)^{2 l}} d \kappa\left|\Psi^{\prime}(x)\right|^{l}+\int_{0}^{1} \frac{\kappa[1-(s \kappa))]}{\left(\kappa \wp_{2}+(1-\kappa) x\right)^{2 l}} d \kappa\left|\Psi^{\prime}\left(\wp_{2}\right)\right|^{l}\right)^{\frac{1}{l}}\right\} \\
& \leqslant \frac{\wp_{1} \wp_{2}}{\wp_{2}-\wp_{1}}\left(\frac{1}{2}\right)^{1-\frac{1}{T}}\left\{\left(x-\wp_{1}\right)^{2}\left(\Lambda_{5}\left|\Psi^{\prime}(x)\right|^{l}+\Lambda_{6}\left|\Psi^{\prime}\left(\wp_{1}\right)\right|^{l}\right)^{\frac{1}{T}}+\left(\wp_{2}-x\right)^{2}\left(\Lambda_{7}\left|\Psi^{\prime}(x)\right|^{l}+\Lambda_{8}\left|\Psi^{\prime}\left(\wp_{2}\right)\right|^{l}\right)^{\frac{1}{T}}\right\} .
\end{aligned}
$$

The proof is complete.

Corollary 2. In Theorem 2, taking $\left|\Psi^{\prime}(x)\right| \leqslant \mathbb{M}, \forall x \in\left[\wp_{1}, \wp_{2}\right]$, we attain the following inequality

$$
\left|\Psi(x)-\frac{\wp_{1} \wp_{2}}{\wp_{2}-\wp_{1}} \int_{\wp_{1}}^{\wp_{2}} \frac{\Psi(u)}{u^{2}} d u\right| \leqslant \frac{\wp_{1} \wp_{2}}{\wp_{2}-\wp_{1}} \mathbb{M}\left(\frac{1}{2}\right)^{1-\frac{1}{l}}\left\{\left(x-\wp_{1}\right)^{2}\left(\Lambda_{5}+\Lambda_{6}\right)^{\frac{1}{T}}+\left(\wp_{2}-x\right)^{2}\left(\Lambda_{7}+\Lambda_{8}\right)^{\frac{1}{T}}\right\} .
$$

Theorem 4. Let $\Psi: \mathbb{A}=\left[\wp_{1}, \wp_{2}\right] \subseteq(0,+\infty) \rightarrow \mathbb{R}$ be a diff mapp on $\mathbb{A}^{0}$, where $\wp_{1}, \wp_{2} \in \mathbb{A}$ with $\wp_{1}<\wp_{2}$ and $\Psi^{\prime} \in L\left[\wp_{1}, \wp_{2}\right]$. If the mapping $\left|\Psi^{\prime}\right|^{l}$ is harmonic s-type convex for $l \geqslant 1$ and $s \in[0,1]$ then for all $x \in\left[\wp_{1}, \wp_{2}\right]$, one has

$$
\begin{aligned}
\mid \Psi(x) & -\frac{\wp_{1} \wp_{2}}{\wp_{2}-\wp_{1}} \int_{\wp_{1}}^{\wp_{2}} \frac{\Psi(u)}{u^{2}} d u \mid \\
\leqslant & \frac{\wp_{1} \wp_{2}}{\wp_{2}-\wp_{1}}\left\{\left(\left(x-\wp_{1}\right)\left\{\frac{1}{\wp_{1}}-\frac{\ln x-\ln \wp_{1}}{x-\wp_{1}}\right\}\right)^{1-\frac{1}{l}}\left(\Lambda_{9}\left|\Psi^{\prime}(x)\right|^{l}+\Lambda_{10}\left|\Psi^{\prime}\left(\wp_{1}\right)\right|^{l}\right)^{\frac{1}{l}}\right. \\
& \left.+\left(\left(\wp_{2}-x\right)\left\{\frac{\ln x-\ln \wp_{1}}{x-\wp_{1}}-\frac{1}{\wp_{2}}\right\}\right)^{1-\frac{1}{T}}\left(\Lambda_{11}\left|\Psi^{\prime}(x)\right|^{l}+\Lambda_{12}\left|\Psi^{\prime}\left(\wp_{2}\right)\right|^{l}\right)^{\frac{1}{T}}\right\}
\end{aligned}
$$

where

$$
\begin{aligned}
& \Lambda_{9}=\frac{\beta(1,1)}{x^{2 l}}{ }_{2} F_{1}\left(2 l, 1 ; 2 ; 1-\frac{\wp_{1}}{x}\right)-s \frac{\beta(1,3)}{x^{2 l}}{ }_{2} F_{1}\left(2 l, 1 ; 3 ; 1-\frac{\wp_{1}}{x}\right), \\
& \Lambda_{10}=\frac{\beta(1,1)}{x^{2 l}}{ }_{2} F_{1}\left(2 l, 1 ; 2 ; 1-\frac{\wp_{1}}{x}\right)-s \frac{\beta(2,1)}{x^{2 l}}{ }_{2} F_{1}\left(2 l, 2 ; 3 ; 1-\frac{\wp_{1}}{x}\right), \\
& \Lambda_{11}=\frac{\beta(1,1)}{x^{2 l}}{ }_{2} F_{1}\left(2 l, 2 ; 3 ; 1-\frac{\wp_{2}}{x}\right)-s \frac{\beta(1,3)}{x^{2 l}}{ }_{2} F_{1}\left(2 l, 1 ; 3 ; 1-\frac{\wp_{2}}{x}\right),
\end{aligned}
$$

and

$$
\Lambda_{12}=\frac{\beta(1,1)}{x^{2 l}}{ }_{2} F_{1}\left(2 l, 2 ; 3 ; 1-\frac{\wp_{2}}{x}\right)-s \frac{\beta(2,1)}{x^{2 l}}{ }_{2} F_{1}\left(2 l, 2 ; 3 ; 1-\frac{\wp_{2}}{x}\right) .
$$


Proof. Applying Lemma 2, properties of modulus, power mean inequality and the property of harmonic s-type convex function of $\left|\Psi^{\prime}\right|^{l}$, we have

$$
\begin{aligned}
& \left|\Psi(x)-\frac{\wp_{1} \wp_{2}}{\wp_{2}-\wp_{1}} \int_{\wp_{1}}^{\wp_{2}} \Psi(u) d u\right| \\
& \leqslant \frac{\wp_{1} \wp_{2}}{\wp_{2}-\wp_{1}}\left\{\left(x-\wp_{1}\right)^{2} \int_{0}^{1} \frac{\kappa}{\left(\kappa \wp_{1}+(1-\kappa) x\right)^{2}}\left|\Psi^{\prime}\left(\frac{\wp_{1} x}{\left(\kappa \wp_{1}+(1-\kappa) x\right)}\right)\right| d \kappa\right. \\
& \left.+\left(\wp_{2}-x\right)^{2} \int_{0}^{1} \frac{\kappa}{\left(\kappa \wp_{2}+(1-\kappa) x\right)^{2}}\left|\Psi^{\prime}\left(\frac{\wp_{2} x}{\left(\kappa \wp_{2}+(1-\kappa) x\right)}\right)\right| d \kappa\right\} \\
& \leqslant \frac{\wp_{1} \wp_{2}\left(x-\wp_{1}\right)^{2}}{\wp_{2}-\wp_{1}}\left(\int_{0}^{1} \frac{\kappa}{\left(\kappa \wp_{1}+(1-\kappa) x\right)^{2}} d \kappa\right)^{1-\frac{1}{T}} \\
& \times\left(\int_{0}^{1} \frac{1}{\left(\kappa \wp_{1}+(1-\kappa) x\right)^{2 l}}\left|\Psi^{\prime}\left(\frac{\wp_{1} x}{\left(\kappa \wp_{1}+(1-\kappa) x\right)}\right)\right|^{l} d \kappa\right)^{\frac{1}{l}} \\
& +\frac{\wp_{1} \wp_{2}\left(\wp_{2}-x\right)^{2}}{\wp_{2}-\wp_{1}}\left(\int_{0}^{1} \frac{\kappa}{\left(\kappa \wp_{2}+(1-\kappa) x\right)^{2}} d \kappa\right)^{1-\frac{1}{l}} \\
& \times\left(\int_{0}^{1} \frac{1}{\left(\kappa \wp_{2}+(1-\kappa) x\right)^{2 l}}\left|\Psi^{\prime}\left(\frac{\wp_{2} x}{\left(\kappa \wp_{2}+(1-\kappa) x\right)}\right)\right|^{l} d \kappa\right)^{\frac{1}{l}} \\
& \leqslant \frac{\wp_{1} \wp_{2}\left(x-\wp_{1}\right)^{2}}{\wp_{2}-\wp_{1}}\left(\frac{1}{x-\wp_{1}}\left\{\frac{1}{\wp_{1}}-\frac{\ln x-\ln \wp_{1}}{x-\wp_{1}}\right\}\right)^{1-\frac{1}{l}} \\
& \times\left(\int_{0}^{1} \frac{\left[[1-s(1-\kappa)]\left|\Psi^{\prime}(x)\right|^{l}+[1-s \kappa]\left|\Psi^{\prime}\left(\wp_{1}\right)\right|^{l}\right]}{\left(\kappa \wp_{1}+(1-\kappa) x\right)^{2 l}} d \kappa\right)^{\frac{1}{l}} \\
& +\frac{\wp_{1} \wp_{2}\left(\wp_{2}-x\right)^{2}}{\wp_{2}-\wp_{1}}\left(\frac{1}{\wp_{2}-x}\left\{\frac{\ln x-\ln \wp_{1}}{x-\wp_{1}}-\frac{1}{\wp_{2}}\right\}\right)^{1-\frac{1}{T}} \\
& \times\left(\int_{0}^{1} \frac{\left[[1-s(1-\kappa)]\left|\Psi^{\prime}(x)\right|^{l}+[1-s \kappa]\left|\Psi^{\prime}\left(\wp_{2}\right)\right|^{l}\right]}{\left(\kappa \wp_{2}+(1-\kappa) x\right)^{2 l}} d \kappa\right)^{\frac{1}{T}} \\
& \leqslant \frac{\wp_{1} \wp_{2}\left(x-\wp_{1}\right)^{2}}{\wp_{2}-\wp_{1}}\left(\frac{1}{x-\wp_{1}}\left\{\frac{1}{\wp_{1}}-\frac{\ln x-\ln \wp_{1}}{x-\wp_{1}}\right\}\right)^{1-\frac{1}{T}} \\
& \times\left(\int_{0}^{1} \frac{[1-s(1-\kappa)]}{\left(\kappa \wp_{1}+(1-\kappa) x\right)^{2 l}} d \kappa\left|\Psi^{\prime}(x)\right|^{l}+\int_{0}^{1} \frac{[1-(s \kappa))]}{\left(\kappa \wp_{1}+(1-\kappa) x\right)^{2 l}} d \kappa\left|\Psi^{\prime}\left(\wp_{1}\right)\right|^{l}\right)^{\frac{1}{l}} \\
& +\frac{\wp_{1} \wp_{2}\left(\wp_{2}-x\right)^{2}}{\wp_{2}-\wp_{1}}\left(\frac{1}{\wp_{2}-x}\left\{\frac{\ln x-\ln \wp_{1}}{x-\wp_{1}}-\frac{1}{\wp_{2}}\right\}\right)^{1-\frac{1}{I}} \\
& \times\left(\int_{0}^{1} \frac{[1-s(1-\kappa)]}{\left(\kappa \wp_{2}+(1-\kappa) x\right)^{2 l}} d \kappa\left|\Psi^{\prime}(x)\right|^{l}+\int_{0}^{1} \frac{[1-(s \kappa))]}{\left(\kappa \wp_{2}+(1-\kappa) x\right)^{2 l}} d \kappa\left|\Psi^{\prime}\left(\wp_{2}\right)\right|^{l}\right)^{\frac{1}{l}} \\
& \leqslant \frac{\wp_{1} \wp_{2}}{\wp_{2}-\wp_{1}}\left\{\left(\left(x-\wp_{1}\right)\left\{\frac{1}{\wp_{1}}-\frac{\ln x-\ln \wp_{1}}{x-\wp_{1}}\right\}\right)^{1-\frac{1}{l}}\left(\Lambda_{9}\left|\Psi^{\prime}(x)\right|^{l}+\Lambda_{10}\left|\Psi^{\prime}\left(\wp_{1}\right)\right|^{l}\right)^{\frac{1}{l}}\right. \\
& \left.+\left(\left(\wp_{2}-x\right)\left\{\frac{\ln x-\ln \wp_{1}}{x-\wp_{1}}-\frac{1}{\wp_{2}}\right\}\right)^{1-\frac{1}{l}}\left(\Lambda_{11}\left|\Psi^{\prime}(x)\right|^{l}+\Lambda_{12}\left|\Psi^{\prime}\left(\wp_{2}\right)\right|^{l}\right)^{\frac{1}{l}}\right\} .
\end{aligned}
$$

The proof is complete.

Corollary 3. In Theorem 4, taking $\left|\Psi^{\prime}(x)\right| \leqslant \mathbb{M}, \forall x \in\left[\wp_{1}, \wp_{2}\right]$, we attain the following inequality

$$
\begin{aligned}
\mid \Psi(x) & -\frac{\wp_{1} \wp_{2}}{\wp_{2}-\wp_{1}} \int_{\wp_{1}}^{\wp_{2}} \frac{\Psi(u)}{u^{2}} d u \mid \\
\leqslant & \frac{\wp_{1} \wp_{2}}{\wp_{2}-\wp_{1}} \mathbb{M}\left\{\left(\left(x-\wp_{1}\right)\left\{\frac{1}{\wp_{1}}-\frac{\ln x-\ln \wp_{1}}{x-\wp_{1}}\right\}\right)^{1-\frac{1}{l}}\left(\Lambda_{9}+\Lambda_{10}\right)^{\frac{1}{l}}\right. \\
& \left.+\left(\left(\wp_{2}-x\right)\left\{\frac{\ln x-\ln \wp_{1}}{x-\wp_{1}}-\frac{1}{\wp_{2}}\right\}\right)^{1-\frac{1}{l}}\left(\Lambda_{11}+\Lambda_{12}\right)^{\frac{1}{l}}\right\} .
\end{aligned}
$$


Theorem 5. Let $\Psi: \mathbb{A}=\left[\wp_{1}, \wp_{2}\right] \subseteq(0,+\infty) \rightarrow \mathbb{R}$ be a diff mapp on $\mathbb{A}^{0}$, where $\wp_{1}, \wp_{2} \in \mathbb{A}$ with $\wp_{1}<\wp_{2}$ and $\Psi^{\prime} \in L\left[\wp_{1}, \wp_{2}\right]$. If the mapping $\left|\Psi^{\prime}\right|^{l}$ is harmonic s-type convex for $q>1, \frac{1}{m}+\frac{1}{l}=1$ and $s \in[0,1]$, then

$$
\begin{aligned}
\left|\Psi(x)-\frac{\wp_{1} \wp_{2}}{\wp_{2}-\wp_{1}} \int_{\wp_{1}}^{\wp_{2}} \frac{\Psi(u)}{u^{2}} d u\right| \leqslant & \frac{\wp_{1} \wp_{2}}{\wp_{2}-\wp_{1}}\left(\frac{1}{m+1}\right)^{\frac{1}{m}}\left\{\left(x-\wp_{1}\right)^{2}\left(\Lambda_{9}\left|\Psi^{\prime}(x)\right|^{l}+\Lambda_{10}\left|\Psi^{\prime}\left(\wp_{1}\right)\right|^{l}\right)^{\frac{1}{T}}\right. \\
& \left.+\left(\wp_{2}-x\right)^{2}\left(\Lambda_{11}\left|\Psi^{\prime}(x)\right|^{l}+\Lambda_{12}\left|\Psi^{\prime}\left(\wp_{2}\right)\right|^{l}\right)^{\frac{1}{T}}\right\}
\end{aligned}
$$

where $\Lambda_{9}, \Lambda_{10}, \Lambda_{11}$ and $\Lambda_{12}$ are defined in Theorem 4 .

Proof. Applying Lemma 2, properties of modulus, Hölder's inequality and the property of harmonic s-type convex function of $\left|\Psi^{\prime}\right|^{l}$, we have

$$
\begin{aligned}
\mid \Psi(x) & -\frac{\wp_{1} \wp_{2}}{\wp_{2}-\wp_{1}} \int_{\wp_{1}}^{\wp_{2}} \Psi(u) d u \mid \\
\leqslant & \frac{\wp_{1} \wp_{2}}{\wp_{2}-\wp_{1}}\left\{\left(x-\wp_{1}\right)^{2} \int_{0}^{1} \frac{\kappa}{\left(\kappa \wp_{1}+(1-\kappa) x\right)^{2}}\left|\Psi^{\prime}\left(\frac{\wp_{1} x}{\left(\kappa \wp_{1}+(1-\kappa) x\right)}\right)\right| d \kappa\right. \\
& \left.+\left(\wp_{2}-x\right)^{2} \int_{0}^{1} \frac{\kappa}{\left(\kappa \wp_{2}+(1-\kappa) x\right)^{2}}\left|\Psi^{\prime}\left(\frac{\wp_{2} x}{\left(\kappa \wp_{2}+(1-\kappa) x\right)}\right)\right| d \kappa\right\} \\
\leqslant & \frac{\wp_{1} \wp_{2}\left(x-\wp_{1}\right)^{2}}{\wp_{2}-\wp_{1}}\left(\int_{0}^{1} \kappa d \kappa\right)^{\frac{1}{m}}\left(\int_{0}^{1} \frac{1}{\left(\kappa \wp_{1}+(1-\kappa) x\right)^{2 l}}\left|\Psi^{\prime}\left(\frac{\wp_{1} x}{\left(\kappa \wp_{1}+(1-\kappa) x\right)}\right)\right|^{l} d \kappa\right)^{\frac{1}{l}} \\
& +\frac{\wp_{1} \wp_{2}\left(\wp_{2}-x\right)^{2}}{\wp_{2}-\wp_{1}}\left(\int_{0}^{1} \kappa d \kappa\right)^{\frac{1}{m}}\left(\int_{0}^{1} \frac{1}{\left(\kappa \wp_{2}+(1-\kappa) x\right)^{2 l}}\left|\Psi^{\prime}\left(\frac{\wp_{2} x}{\left(\kappa \wp_{2}+(1-\kappa) x\right)}\right)\right|^{l} d \kappa\right)^{\frac{1}{l}} \\
\leqslant & \frac{\wp_{1} \wp_{2}\left(x-\wp_{1}\right)^{2}}{\wp_{2}-\wp_{1}}\left(\frac{1}{m+1}\right)^{\frac{1}{m}}\left(\int_{0}^{1} \frac{\left[[1-s(1-\kappa)]\left|\Psi^{\prime}(x)\right|^{l}+[1-s \kappa]\left|\Psi^{\prime}\left(\wp_{1}\right)\right|^{l}\right]}{\left(\kappa \wp_{1}+(1-\kappa) x\right)^{2 l}} d \kappa\right)^{\frac{1}{T}} \\
& +\frac{\wp_{1} \wp_{2}\left(\wp_{2}-x\right)^{2}}{\wp_{2}-\wp_{1}}\left(\frac{1}{m+1}\right)^{\frac{1}{m}}\left(\int_{0}^{1} \frac{\left[[1-s(1-\kappa)]\left|\Psi^{\prime}(x)\right|^{l}+[1-s \kappa]\left|\Psi^{\prime}\left(\wp_{2}\right)\right|^{l}\right]}{\left(\kappa \wp_{2}+(1-\kappa) x\right)^{2 l}} d \kappa\right)^{\frac{1}{l}} \\
\leqslant & \frac{\wp_{1} \wp_{2}}{\wp_{2}-\wp_{1}}\left(\frac{1}{m+1}\right)^{\frac{1}{m}}\left\{\left(x-\wp_{1}\right)^{2}\left(\Lambda_{9}\left|\Psi^{\prime}(x)\right|^{l}+\Lambda_{10}\left|\Psi^{\prime}\left(\wp_{1}\right)\right|^{l}\right)^{\frac{1}{l}}\right. \\
& \left.+\left(\wp_{2}-x\right)^{2}\left(\Lambda_{11}\left|\Psi^{\prime}(x)\right|^{l}+\Lambda_{12}\left|\Psi^{\prime}\left(\wp_{2}\right)\right|^{l}\right)^{\frac{1}{l}}\right\} .
\end{aligned}
$$

The proof is complete.

Corollary 4. In Theorem 5, taking $\left|\Psi^{\prime}(x)\right| \leqslant \mathbb{M}, \forall x \in\left[\wp_{1}, \wp_{2}\right]$, we attain the following inequality

$$
\left|\Psi(x)-\frac{\wp_{1} \wp_{2}}{\wp_{2}-\wp_{1}} \int_{\wp_{1}}^{\wp_{2}} \frac{\Psi(u)}{u^{2}} d u\right| \leqslant \frac{\wp_{1} \wp_{2}}{\wp_{2}-\wp_{1}} \mathbb{M}\left(\frac{1}{m+1}\right)^{\frac{1}{m}}\left\{\left(x-\wp_{1}\right)^{2}\left(\Lambda_{9}+\Lambda_{10}\right)^{\frac{1}{T}}+\left(\wp_{2}-x\right)^{2}\left(\Lambda_{11}+\Lambda_{12}\right)^{\frac{1}{T}}\right\} .
$$

Theorem 6. Let $\Psi: \mathbb{A}=\left[\wp_{1}, \wp_{2}\right] \subseteq(0,+\infty) \rightarrow \mathbb{R}$ be a diff mapp on $\mathbb{A}^{0}$, where $\wp_{1}, \wp_{2} \in \mathbb{A}$ with $\wp_{1}<\wp_{2}$ and $\Psi^{\prime} \in L\left[\wp_{1}, \wp_{2}\right]$. If the mapping $\left|\Psi^{\prime}\right|^{l}$ is harmonic s-type convex for $l>1, \frac{1}{m}+\frac{1}{l}=1$ and $s \in[0,1]$, then

$$
\begin{aligned}
\mid \Psi(x) & -\frac{\wp_{1} \wp_{2}}{\wp_{2}-\wp_{1}} \int_{\wp_{1}}^{\wp_{2}} \frac{\Psi(u)}{u^{2}} d u \mid \\
\leqslant & \frac{\wp_{1} \wp_{2}}{\wp_{2}-\wp_{1}}\left(\left[\frac{2-s}{2}\right]\right)^{\frac{1}{T}}\left\{\left(x-\wp_{1}\right)^{2}\left(\frac{\beta(m+1,1)}{x^{2 m}}{ }_{2} F_{1}\left(2 m, m+1, m+2,1-\frac{\wp_{1}}{x}\right)\right)\left(\left\{\left|\Psi^{\prime}(x)\right|^{l}+\left|\Psi^{\prime}\left(\wp_{1}\right)\right|^{l}\right\}\right)^{\frac{1}{T}}\right. \\
& \left.+\left(\wp_{2}-x\right)^{2}\left(\frac{\beta(1,1+m)}{\wp_{2}^{2 m}}{ }_{2} F_{1}\left(2 m, 1 ; m+2,1-\frac{x}{\wp_{2}}\right)\right)\left(\left\{\left|\Psi^{\prime}(x)\right|^{l}+\left|\Psi^{\prime}\left(\wp_{2}\right)\right|^{l}\right\}\right)^{\frac{1}{T}}\right\} .
\end{aligned}
$$


Proof. Applying Lemma 2, properties of modulus, Hölder's inequality and the property of harmonic s-type convex function of $\left|\Psi^{\prime}\right|^{l}$, we have

$$
\begin{aligned}
& \left|\Psi(x)-\frac{\wp_{1} \wp_{2}}{\wp_{2}-\wp_{1}} \int_{\wp_{1}}^{\wp_{2}} \Psi(u) d u\right| \\
& \leqslant \frac{\wp_{1} \wp_{2}}{\wp_{2}-\wp_{1}}\left\{\left(x-\wp_{1}\right)^{2} \int_{0}^{1} \frac{\kappa}{\left(\kappa \wp_{1}+(1-\kappa) x\right)^{2}}\left|\Psi^{\prime}\left(\frac{\wp_{1} x}{\left(\kappa \wp_{1}+(1-\kappa) x\right)}\right)\right| d \kappa\right. \\
& \left.+\left(\wp_{2}-x\right)^{2} \int_{0}^{1} \frac{\kappa}{\left(\kappa \wp_{2}+(1-\kappa) x\right)^{2}}\left|\Psi^{\prime}\left(\frac{\wp_{2} x}{\left(\kappa \wp_{2}+(1-\kappa) x\right)}\right)\right| d \kappa\right\} \\
& \leqslant \frac{\wp_{1} \wp_{2}\left(x-\wp_{1}\right)^{2}}{\wp_{2}-\wp_{1}}\left(\int_{0}^{1} \frac{\kappa^{m}}{\left(\kappa \wp_{1}+(1-\kappa) x\right)^{2}} d \kappa\right)^{\frac{1}{m}}\left(\int_{0}^{1}\left|\Psi^{\prime}\left(\frac{\wp_{1} x}{(\kappa a+(1-\kappa) x)}\right)\right|^{l} d \kappa\right)^{\frac{1}{l}} \\
& +\frac{\wp_{1} \wp_{2}\left(\wp_{2}-x\right)^{2}}{\wp_{2}-\wp_{1}}\left(\int_{0}^{1} \frac{\kappa^{m}}{\left(\kappa \wp_{2}+(1-\kappa) x\right)^{2}} d \kappa\right)^{\frac{1}{m}}\left(\int_{0}^{1}\left|\Psi^{\prime}\left(\frac{\wp_{2} x}{\left(\kappa \wp_{2}+(1-\kappa) x\right)}\right)\right|^{l} d \kappa\right)^{\frac{1}{T}} \\
& \leqslant \frac{\wp_{1} \wp_{2}\left(x-\wp_{1}\right)^{2}}{\wp_{2}-\wp_{1}}\left(\frac{\beta(m+1,1)}{x^{2 m}}{ }_{2} F_{1}\left(2 m, m+1, m+2,1-\frac{\wp_{1}}{x}\right)\right) \\
& \times\left(\int_{0}^{1}\left[[1-s(1-\kappa)]\left|\Psi^{\prime}(x)\right|^{l}+[1-s \kappa]\left|\Psi^{\prime}\left(\wp_{1}\right)\right|^{l}\right] d \kappa\right)^{\frac{1}{T}} \\
& +\frac{\wp_{1} \wp_{2}\left(\wp_{2}-x\right)^{2}}{\wp_{2}-\wp_{1}}\left(\frac{\beta(1,1+m)}{\wp_{2}^{2 m}}{ }_{2} F_{1}\left(2 m, 1 ; m+2,1-\frac{x}{\wp_{2}}\right)\right) \\
& \times\left(\int_{0}^{1}\left[[1-s(1-\kappa)]\left|\Psi^{\prime}(x)\right|^{l}+[1-s \kappa]\left|\Psi^{\prime}\left(\wp_{2}\right)\right|^{l}\right] d k\right)^{\frac{1}{l}} \\
& \leqslant \frac{\wp_{1} \wp_{2}\left(x-\wp_{1}\right)^{2}}{\wp_{2}-\wp_{1}}\left(\frac{\beta(m+1,1)}{x^{2 m}}{ }_{2} F_{1}\left(2 m, m+1, m+2,1-\frac{\wp_{1}}{x}\right)\right)\left(\left[\frac{2-s}{2}\right]\left\{\left|\Psi^{\prime}(x)\right|^{l}+\left|\Psi^{\prime}\left(\wp_{1}\right)\right|^{l}\right\}\right)^{\frac{1}{T}} \\
& +\frac{\wp_{1} \wp_{2}\left(\wp_{2}-x\right)^{2}}{\wp_{2}-\wp_{1}}\left(\frac{\beta(1,1+m)}{\wp_{2}^{2 m}}{ }_{2} F_{1}\left(2 m, 1 ; m+2,1-\frac{x}{\wp_{2}}\right)\right)\left(\left[\frac{2-s}{2}\right]\left\{\left|\Psi^{\prime}(x)\right|^{l}+\left|\Psi^{\prime}\left(\wp_{2}\right)\right|^{l}\right\}\right)^{\frac{1}{T}} \\
& \leqslant \frac{\wp_{1} \wp_{2}}{\wp_{2}-\wp_{1}}\left(\left[\frac{2-s}{2}\right]\right)^{\frac{1}{l}}\left\{\left(x-\wp_{1}\right)^{2}\left(\frac{\beta(m+1,1)}{x^{2 m}}{ }_{2} F_{1}\left(2 m, m+1, m+2,1-\frac{\wp_{1}}{x}\right)\right)\left(\left\{\left|\Psi^{\prime}(x)\right|^{l}+\left|\Psi^{\prime}\left(\wp_{1}\right)\right|^{l}\right\}\right)^{\frac{1}{l}}\right. \\
& \left.+\left(\wp_{2}-x\right)^{2}\left(\frac{\beta(1,1+m)}{\wp_{2}^{2 m}}{ }_{2} F_{1}\left(2 m, 1 ; m+2,1-\frac{x}{\wp_{2}}\right)\right)\left(\left\{\left|\Psi^{\prime}(x)\right|^{l}+\left|\Psi^{\prime}\left(\wp_{2}\right)\right|^{l}\right\}\right)^{\frac{1}{l}}\right\} \text {. }
\end{aligned}
$$

The proof is complete.

Corollary 5. In Theorem 6, taking $\left|\Psi^{\prime}(x)\right| \leqslant \mathbb{M}, \forall x \in\left[\wp_{1}, \wp_{2}\right]$, we attain the following inequality

$$
\begin{aligned}
\mid \Psi(x) & -\frac{\wp_{1} \wp_{2}}{\wp_{2}-\wp_{1}} \int_{\wp_{1}}^{\wp_{2}} \frac{\Psi(u)}{u^{2}} d u \mid \\
\leqslant & \frac{\wp_{1} \wp_{2}}{\wp_{2}-\wp_{1}} 2 \mathbb{M}\left(\left[\frac{2-s}{2}\right]\right)^{\frac{1}{T}}\left\{\left(x-\wp_{1}\right)^{2}\left(\frac{\beta(m+1,1)}{x^{2 m}}{ }_{2} F_{1}\left(2 m, m+1, m+2,1-\frac{\wp_{1}}{x}\right)\right)\right. \\
& \left.+\left(\wp_{2}-x\right)^{2}\left(\frac{\beta(1,1+m)}{\wp_{2}^{2 m}}{ }_{2} F_{1}\left(2 m, 1 ; m+2,1-\frac{x}{\wp_{2}}\right)\right)\right\} .
\end{aligned}
$$

Remark 2. In the previous inequalities, by taking $x=\frac{\wp_{1}+\wp_{2}}{2}$, we attain multiple midpoint type inequalities.

\section{Conclusion}

In this work, we defined harmonic s-type convex functions and obtained the refinements of Ostrowski type inequality. The intriguing procedure and amusing ideas of this work can be extended on the coordinates and strongly harmonic s-type convex functions along with fractional integral calculus.

Acknowledgments: Authors are thankful to the anonymous referee for his constructive comments and feedback.

Author Contributions: All authors contributed equally to the writing of this paper. All authors read and approved the final manuscript. 
Conflicts of Interest: "The authors declare no conflict of interest".

\section{References}

[1] Xi, B. Y., \& Qi, F. (2012). Some integral inequalities of Hermite-Hadamard type for convex functions with applications to means. Journal of Function Spaces and Applications, 2012, Article ID 980438.

[2] Özcan, S., \& İşcan, İ. (2019). Some new Hermite-Hadamard type integral inequalities for the $s$-convex functions and theirs applications. Journal of Inequalities and Applications, 2019, Artilce No. 201.

[3] Butt, S. I., Kashuri, A., Tariq, M., Nasir, J., Aslam, A., \& Gao, W. (2020). n-polynomial exponential type $p$-convex function with some related inequalities and their applications. Heliyon, 6(11), e05420.

[4] Butt, S. I., Tariq, M., Aslam, A., Ahmad, H., \& Nofal, T. A. (2021). Hermite-hadamard type inequalities via generalized harmonic exponential convexity and applications. Journal of Function Spaces, 2021, Article ID 5533491.

[5] Butt, S. I., Nadeem, M., Qaisar, S., Akdemir, A. O., \& Abdeljawad, T. (2020). Hermite-Jensen-Mercer type inequalities for conformable integrals and related results. Advances in Difference Equations, 2020, Artilce No. 501.

[6] Ostrowski, A. (1938). Über die Absolutabweichung einer differentiebaren funktion von ihren integralmittelwert. Comment. Math. Helv., 10, 226-227.

[7] Milovanović, G. V., \& Peçarić, J. E. (1976). On generalization of the inequality of A. Ostrowski and some related applications. Publikacije Elektrotehnickog fakulteta. Serija Matematika i fizika, (544/576), 155-158.

[8] Milovanović, G. V. (1975). On some integral inequalities. Publikacije Elektrotehnickog fakulteta. Serija Matematika $i$ fizika, (498/541), 119-124.

[9] Dragomir, S. S., \& Wang, S. (1997). An inequality of Ostrowski-Grs̈s type and its applications to the estimation of error bounds for some special means and for some numerical quadrature rules. Computers $\mathcal{E}$ Mathematics with Applications, 33(11), 15-20.

[10] Cerone, P., \& Dragomir, S. S. (2004). Ostrowski type inequalities for functions whose derivatives satisfy certain convexity assumptions. Demonstratio Mathematica, 37(2), 299-308.

[11] Noor, M. A., Noor, K. I., \& Awan, M. U. (2014). Fractional Ostrowski inequalities for s-Godunova-Levin functions. International Journal of Analysis and Applications, 5(2), 167-173.

[12] Set, E. (2012). New inequalities of Ostrowski type for mappings whose derivatives are s-convex in the second sense via fractional integrals. Computers $\mathcal{E}$ Mathematics with Applications, 63(7), 1147-1154.

[13] Niculescu, C., \& Persson, L. E. (2006). Convex Functions and their Applications (pp. xvi+-255). New York: Springer.

[14] Shi, H. N., \& Zhang, J. (2013). Some new judgement theorems of Schur geometric and Schur harmonic convexities for a class of symmetric functions. Journal of Inequalities and Applications, 2013, Article No. 527.

[15] İşcan, İ. (2014). Hermite-Hadamard type inequalities for harmonically convex functions. Hacettepe Journal of Mathematics and statistics, 43(6), 935-942.

[16] Rashid, S., İşcan, İ., Baleanu, D., \& Chu, Y. M. (2020). Generation of new fractional inequalities via n-polynomials s-type convexity with applications. Advances in Difference Equations, 2020, Article No. 264.

[17] Sommerfeld, A. (1949). Partial Differential Equations in Physics. Academic press.

[18] Françoise, J. P., Naber, G. L., \& Tsou, S. T. (Eds.). (2006). Encyclopedia of Mathematical Physics (Vol. 2, pp. p-189). Amsterdam: Elsevier.

[19] Abramowitz, M., \& Stegun, I. A. (1965). Handbook of Mathematical Functions with Formulas, Graphs, and Mathematical Tables. Dover, New York.

[20] Baloch, I. A., De La Sen, M., \& İşcan, İ. (2019). Characterizations of classes of harmonic convex functions and applications. International Journal of Analysis and Applications, 17(5), 722-733.

[21] İşcan, İ. (2015). Some Ostrowski type inequalities for harmonically (s)-convex functions. Konuralp Journal of Mathematics, 3(1) (2015), 63-74. 